\title{
La promoción de la marca hospitalaria a través de la comunicación interpersonal que mantiene el profesional de la salud en las redes sociales
}

\section{Hospital Brand Promotion Through Interpersonal Communication Maintained by Health Professionals on Social Media}

Pablo Medina Aguerrebere

Canadian University Dubai (Emiratos Árabes)

La implantación de las redes sociales como una herramienta de comunicación institucional, las nuevas exigencias de los pacientes y la mayor competencia entre hospitales han obligado a estas organizaciones a repensar sus estrategias de comunicación. La creación de una marca reputada que ayude a consolidar el posicionamiento estratégico de la organización en el mercado sanitario se ha convertido en una prioridad estratégica para todos los hospitales. El objetivo de este artículo de revisión bibliográfica es analizar el impacto que tiene en la construcción de la marca hospitalaria la comunicación interpersonal que el profesional de la salud mantiene en las redes sociales con los stakeholders del hospital. El artículo propone un modelo de promoción de marca basado en la comunicación interpersonal que realiza este profesional en las redes sociales y concluye que los hospitales que desean construir una
The implementation of social media as a corporate communication tool, the new demands of patients, and greater competition between hospitals have led these organizations to rethink their communication strategies. The creation of a reputed brand that helps an organization to reinforce its strategic positioning in the health market has become a strategic priority for all hospitals. This literature review paper aims to analyze the impact on hospital brand construction of the interpersonal communication between health professionals and the organization's stakeholders in social media. This paper proposes a model of brand building based on the interpersonal communication carried out by these professionals in social media, and concludes that hospitals that want to build a reputed brand must take economic and management measures, implement communication plans and 
marca reputada deben tomar medidas económicas y de management, instaurar planes de comunicación y fomentar la colaboración entre profesionales de la salud y de la comunicación.

Palabras clave: hospital, marca, comunicación interpersonal, profesional de la salud, redes sociales. promote collaboration between health professionals and corporate communication experts.

Keywords: hospital, brand, interpersonal communication, health professionals, social media.

$\mathrm{L}$ as organizaciones hospitalarias apuestan cada vez más por la comunicación institucional como una herramienta estratégica capaz de influenciar de manera positiva en el desarrollo de la organización. Desde hace unos años, diversos factores han llevado a estas organizaciones a repensar sus estrategias de comunicación institucional: el rol más activo desempeñado por el paciente, el desarrollo de las redes sociales, la competencia creciente entre hospitales públicos y privados, el deseo de la población en formarse en temas médicos y de prevención, etc. Estas organizaciones apuestan por la comunicación institucional como método para crear una marca sólida que les ayude a mantener relaciones satisfactorias con los distintos stakeholders, como por ejemplo los pacientes, los medios de comunicación o las autoridades públicas. Sin embargo, el marco legal, las connotaciones vitales del servicio médico propuesto por los hospitales, la dificultad para divulgar conceptos científicos y la escasa tradición comunicativa de estas organizaciones dificultan la implementación en el hospital de una gestión profesional de la comunicación institucional.

El objetivo de este artículo es analizar el rol que juegan los profesionales de la salud - personal médico y de enfermería - en la creación de una marca hospitalaria sólida, así como también el impacto que tienen en la marca hospitalaria las acciones de comunicación interpersonal que llevan a cabo los profesionales de la salud en las redes sociales gestionadas por el hospital. Para ello, se realiza, en primer lugar, una revisión bibliográfica sobre los conceptos de comunicación interpersonal, comunicación interna, comunicación externa, marca hospitalaria y redes sociales; y, en segundo lugar, antes de las conclusiones, se propone un modelo de comunicación de marca basado en la comunicación interpersonal que realizan los profesionales de la salud en las redes sociales.

\section{LA MARCA HOSPITALARIA}

La gestión profesional de la comunicación institucional se ha convertido en una prioridad estratégica para todos los hospitales que desean construir una mar- 
ca sólida y reputada que les permita establecer relaciones satisfactorias con sus principales stakeholders — pacientes, autoridades públicas, etc. - y así reforzar su posicionamiento estratégico en el mercado sanitario (Medina, 2017a). Para ello, dichas organizaciones necesitan crear un departamento de comunicación que se encargue de gestionar esta actividad de un modo integrado y profesional para evitar así la duplicación de esfuerzos y garantizar el impacto positivo de la comunicación en el funcionamiento del hospital (Maier, 2016). Dicho departamento debe contar con diferentes profesionales - periodistas, gestores, médicos, enfermeros, economistas, etc.- que sean capaces de gestionar el proceso comunicativo hospitalario de un modo global y multidisciplinar (Hannawa et al., 2015). El director de dicho departamento se encarga de liderar tres actividades principales: a) la comunicación interpersonal, b) la comunicación interna y c) la comunicación externa.

a) La comunicación interpersonal. La organización de cursos para formar al profesional de la salud en las habilidades de comunicación interpersonal contribuye a mejorar el funcionamiento del hospital, así como la satisfacción del paciente con el servicio médico ofrecido (Gilligan et al., 2016). La capacidad de este profesional para difundir claramente conceptos científicos, gestionar el silencio durante las consultas con el paciente, implicar a este último en el diseño del tratamiento médico y mostrarle empatía y comprensión influye positivamente en la imagen que el paciente tiene sobre el profesional de la salud y sobre el centro hospitalario (Al-Abri y Al-Balushi, 2014). Además, el departamento de comunicación debe proponer al profesional de la salud cursos sobre comunicación multicultural que le permitan desarrollar las competencias necesarias para adaptar su discurso y su comportamiento a pacientes de diferentes culturas, idiomas o religiones (Weech et al., 2012).

b) La comunicación interna. La gestión estratégica de esta actividad tiene un doble impacto positivo en el hospital: por un lado, contribuye a mejorar el funcionamiento interno de la organización, el trabajo en equipo entre los empleados, la gestión de la información médica y la satisfacción del paciente (Medina, 2011); y, por otro, influye positivamente en la imagen que el empleado tiene sobre la organización, lo cual ayuda a reforzar su compromiso con la misma y, por tanto, a fomentar la reputación de marca del hospital (Bourke, 2009). El director de comunicación del hospital debe evaluar constantemente los cambios internos y externos a los que está sometida la organización para así instaurar iniciativas de comunicación interna adaptadas e innovadoras (Pelitti, 2016).

c) La comunicación externa. Los hospitales interactúan con un gran número de stakeholders, como por ejemplo las autoridades sanitarias, los medios de comunicación, los laboratorios farmacéuticos, las compañías de seguros y, sobre todo, los pacientes y sus familiares (Medina, 2011). Antes de implementar la estrategia de comunicación externa, el director de comunicación debe analizar los objetivos globales perseguidos por la organización, el comportamiento actual de la competencia, las tendencias existentes en el sector, así como los cambios de actitud en los distintos stakeholders (Moser y Greeman, 2014). Durante la implementación de la estrategia de comunicación externa, y sobre todo después de la misma, el 
director de comunicación recurre a los indicadores de evaluación previamente definidos para analizar el impacto de todas y cada una de las acciones en la reputación de la organización, la imagen de marca, las ventas e incluso la motivación de los empleados y de los stakeholders (Zerfass y Viertmann, 2017).

La implementación de iniciativas de comunicación interpersonal, interna y externa tiene como objetivo principal promover la imagen de marca del hospital entre sus distintos stakeholders (Medina, 2011). La marca corporativa representa distintas cualidades tangibles e intangibles que aportan a la organización un valor añadido, el cual influye positivamente en la imagen que los stakeholders tienen sobre la organización (Esposito, 2017). En el caso de un hospital, la marca no hace referencia únicamente al nombre de la organización, sino también a todas las experiencias vividas por el paciente cuando acude al hospital, así como al cumplimiento - o no- de las expectativas que este tenía sobre la promesa corporativa de la organización (Wang et al., 2011). La competencia creciente entre las organizaciones hospitalarias, así como las nuevas exigencias de los pacientes, obligan a los hospitales a gestionar estratégicamente su imagen de marca, lo que implica, entre otros aspectos, identificar distintos indicadores objetivos que permitan evaluar de un modo cuantitativo el impacto de la marca en el funcionamiento de la organización (Veltri y Nardo, 2013).

Los cinco elementos intangibles que constituyen la arquitectura de marca de un hospital son la identidad, los valores, la misión, la visión y la cultura corporativa. La identidad hace referencia a la esencia de la organización, es decir, las razones que llevaron a los fundadores de la organización a crear el hospital (Medina, 2011). Aunque la definición de la identidad la realiza el departamento de comunicación, la difusión de la misma concierne a todos los empleados del hospital. La identidad se plasma en realidades más concretas y tangibles denominadas "valores", que ayudan a los empleados a realizar su trabajo en el día a día (Sheehan e Isaac, 2014). En función de la identidad y de los valores, el hospital define su misión, la cual hace referencia a los objetivos perseguidos por la organización en el medio plazo; no se trata únicamente de una formulación estratégica, sino de una directiva que debe ser lo suficientemente clara como para que los empleados la apliquen en la práctica (Cady et al., 2011). Por su parte, la visión establece los objetivos de largo plazo que persigue la organización, así como los cambios necesarios que esta debe llevar a cabo para alcanzar dichos objetivos (Singal y Jain, 2013). Finalmente, la cultura corporativa hace referencia a la manera única de trabajar que tienen los empleados de una organización; dicha cultura queda determinada por la capacidad del hospital para alinear la misión, los valores y las prácticas profesionales de cada día (Nelson, Taylor y Walsh, 2014).

La gestión estratégica de la comunicación interpersonal, interna y externa como herramientas para difundir la arquitectura de marca del hospital contribuye positivamente a reforzar la reputación de la organización hospitalaria ante sus distintos stakeholders. Los hospitales que gozan de una buena reputación transmiten una imagen de profesionalidad y seguridad en el tratamiento ofrecido a los pacientes (Mira, Lorenzo y Navarro, 2014). Para construir una marca reputada, el director de comunicación del hospital debe implantar iniciativas de comunicación que realcen el valor del profesional de la salud y que mejoren la 
satisfacción del paciente (Torres, Vásquez y Barra, 2009). Además, debe analizar constantemente la opinión de los stakeholders — especialmente los pacientessobre la organización para así adaptar de un modo coherente las estrategias de comunicación y alcanzar una mayor efectividad (Gay y Pho, 2013).

\section{LA MARCA HOSPITALARIA EN LAS REDES SOCIALES}

En el entorno hospitalario, las redes sociales se han convertido en una herramienta de comunicación institucional muy utilizada por todas aquellas organizaciones que desean dinamizar su comunicación de marca y establecer relaciones satisfactorias con los distintos stakeholders. Si bien es cierto que muchos centros hospitalarios se están incorporando tardíamente a esta dinámica de redes sociales (Fernández y Bau, 2015) y que algunos de ellos todavía no han conseguido resolver distintos problemas relativos a la confidencialidad en el uso de la información, la gestión de la identidad del paciente y la participación activa del profesional de la salud en la comunicación online (Rando y Heras, 2016), las redes sociales han cambiado radicalmente las estrategias de comunicación institucional utilizadas por la mayor parte de los hospitales. La capacidad de estas plataformas para establecer y potenciar relaciones de confianza con los stakeholders de la organización las ha convertido en la herramienta comunicativa más utilizada por las organizaciones sanitarias, por delante de los medios tradicionales como la prensa, la televisión o la radio (Griffis et al., 2014).

Las redes sociales ayudan a los hospitales a dinamizar su marca y a mejorar su reputación ante los distintos stakeholders. Existen diez razones por las cuales se puede realizar esta afirmación. En primer lugar, gracias a las redes sociales, los hospitales pueden acceder a mucha información relativa al paciente (quejas, expectativas, sugerencias, etc.), lo que constituye una ayuda importante para que el profesional de la salud realice mejor su trabajo y así se mejore la satisfacción del paciente con la marca hospitalaria (Bubien, 2015). En segundo lugar, las redes sociales son de una gran utilidad para implantar en el hospital un sistema de toma de decisiones colectivas entre el profesional de la salud y el paciente; dicho sistema influye positivamente en el compromiso del paciente con el tratamiento médico establecido, así como en su satisfacción con la organización hospitalaria en su conjunto (Lim, 2016). En tercer lugar, el dialogo bidireccional y constante que facilitan estas plataformas constituye una oportunidad para que los hospitales divulguen conceptos científicos de un modo ameno, profesional, ético y preciso; dicho de otro modo, gracias a las redes sociales, los hospitales pueden aportar al paciente un valor añadido en forma de contenido científico de calidad (Chretien y Terry, 2013). En cuarto lugar, las redes sociales gestionadas por la organización ofrecen al hospital la posibilidad de controlar las conversaciones públicas que mantienen sus profesionales de la salud con los pacientes para así garantizar la precisión científica de los comentarios difundidos, lo que influye positivamente en la credibilidad de la marca hospitalaria como fuente de información médica (Abramson, Keefe y Chou, 2015). En quinto lugar, la pluralidad de formatos que proponen las redes sociales (videos, audios, infografías, etc.) contribuye positivamente a generar en el paciente un interés marcado por los temas de educación 
en salud y prevención (Gabarrón et al., 2013). En sexto lugar, las redes sociales ayudan a las organizaciones a instaurar una estrategia de comunicación basada en el dialogo, la participación y la difusión de contenido constructivo sin carácter comercial, lo que constituye una oportunidad para que el hospital pueda realizar, junto con sus stakeholders, una construcción colectiva de su marca (Blomgren, Hedmo y Waks, 2016). En séptimo lugar, gracias a las redes sociales, los pacientes pueden comunicarse directamente con los profesionales de la salud que trabajan en el hospital y establecer con ellos una relación más humana y emocional, lo que influye positivamente en las percepciones que tienen sobre la marca hospitalaria (Medina, 2011). En octavo lugar, la versatilidad ofrecida por las redes sociales permite a las organizaciones concebir e instaurar estrategias de comunicación personalizadas que consigan satisfacer las diferentes necesidades y expectativas de todos y cada uno de los stakeholders (McCarroll et al., 2014). En noveno lugar, todos los empleados del hospital, especialmente los profesionales de la salud, pueden utilizar dichas herramientas para divulgar información institucional (eventos, congresos, etc.), lo que permite activar un boca a oreja muy efectivo para mejorar la visibilidad de la marca hospitalaria (McCarroll et al., 2014). Y, en décimo lugar, las redes sociales ofrecen a los hospitales diferentes indicadores para evaluar el impacto que generan en la reputación del hospital las distintas informaciones divulgadas, lo que ayuda al hospital a adaptar su mensaje constantemente para así lograr una mayor eficacia comunicativa (McCaughey et al., 2014).

Las organizaciones hospitalarias están sometidas al cambio constante: desarrollo tecnológico, creación de nuevos tratamientos médicos, aparición de nuevos competidores, etc. (Kemp, Jillapalli y Becerra, 2014). Esta situación también concierne a los pacientes, quienes no solo reciben cada vez más información y disponen de un mejor acceso a los servicios sanitarios propuestos por diferentes organizaciones, sino que además están muy influenciados por la opinión de otros pacientes (Gay y Pho, 2013). En este contexto de cambio constante, resulta necesario que las organizaciones hospitalarias desarrollen estrategias de comunicación de marca muy potentes para potenciar así la imagen del hospital ante los distintos stakeholders y prevenir así posibles crisis de reputación (Kemp, Jillapalli y Becerra, 2014). Y para ello, los hospitales necesitan contar con la participación activa de los profesionales de la salud en las redes sociales que dependen del hospital: dichos profesionales deben convertirse en los mejores comunicadores del hospital, en verdaderos embajadores de marca capaces de divulgar información científica/institucional y generar compromiso en el paciente para así influenciar sus percepciones sobre la organización.

\section{EL ROL DEL MÉDICO EN LA PROMOCIÓN DE LA MARCA HOSPITALARIA 2.0}

La consolidación de las redes sociales como una herramienta de comunicación institucional impacta positivamente en el trabajo desarrollado por el profesional de la salud, no solo desde un punto de vista comunicativo sino también clínico (Medina, 2017b). Los profesionales de la comunicación institucional se han convertido en verdaderos aliados de los profesionales de la salud, ya que les ayudan a utilizar correctamente dichas plataformas para que impacten positivamente en la 
satisfacción del paciente con el servicio médico ofrecido (Ruiz, 2015). La colaboración entre ambos profesionales tiene como objetivo la elaboración y difusión de información científica que ayude al paciente a comprender mejor su patología y su tratamiento, pero que impacte también positivamente en la imagen que el paciente tiene sobre el hospital (McCarroll et al., 2014). Dicha colaboración se plasma, entre otros aspectos, en la creación de manuales de comunicación online que guían el comportamiento del profesional de la salud en las redes sociales, de tal modo que dicho comportamiento ayude al hospital a alcanzar sus objetivos estratégicos, ya sean estos comunicativos, científicos o de desarrollo organizacional (Farnan et al., 2013). La protocolización de las actividades comunicativas que el profesional de la salud lleva a cabo en las redes sociales es necesaria para garantizar que la imagen transmitida a los diferentes stakeholders sea unívoca y coherente con la imagen que el hospital transmite a través del resto de iniciativas de comunicación institucional —internas y externas- (Würz, Nurm y Ekdahl, 2013). Cuando los profesionales de la salud utilizan las redes sociales, deben respetar cuatro principios: a) mantener la profesionalidad en todo momento, $b$ ) implicarse en la comunicación e intentar satisfacer las necesidades informativas de los usuarios, $c$ ) solicitar ayuda al departamento de comunicación siempre que sea necesario y $d$ ) difundir información que refuerce el compromiso del usuario con el hospital (Grajales et al., 2014).

La participación del profesional de la salud en las redes sociales tiene como objetivo ayudar al hospital a promocionar la marca corporativa, pero también debe contribuir a construir y fomentar la propia marca del profesional de la salud. El personal branding de un profesional de la salud puede ser definido como el valor profesional y comunicativo que este aporta a la organización (Trepanier y Gooch, 2014). En el contexto hospitalario, para construir una marca personal reputada, resulta necesario que el departamento de comunicación diseñe y ejecute un plan de comunicación institucional adaptado a cada profesional de la salud; dicho plan debe considerar varios aspectos como, por ejemplo, las expectativas del empleado, las exigencias de los stakeholders, las tácticas comunicativas necesarias para construir el reconocimiento de marca y las ventajas tangibles que la marca personal puede aportar al profesional de la salud (Trepanier y Goodch, 2014). Desde un punto de vista comunicativo, dicho profesional se ha convertido en una submarca del hospital que el departamento de comunicación debe gestionar de un modo estratégico para que su comportamiento en las redes sociales refuerce la marca global del hospital, así como la propia marca personal. La reputación del profesional de la salud impacta positivamente en la reputación del hospital: las dos marcas se nutren y complementan mutuamente, de ahí la importancia de que ambas sean gestionadas de un modo estratégico por profesionales de la comunicación (Torres, Vásquez y Barra, 2009).

Uno de los protagonistas principales de la comunicación online de los hospitales es el director de comunicación. Este profesional de la comunicación se encarga de gestionar de un modo estratégico todas y cada una de las iniciativas de comunicación —interna, externa - con el objetivo de aportar valor a la marca e influenciar positivamente en el funcionamiento de la organización (Zerfass y Viertmann, 2017). El director de comunicación forma parte del consejo de 
16 administración del hospital, lo que le permite tener una visión integral de las decisiones que lleva a cabo la organización así como del impacto comunicativo de dichas decisiones (Medina, 2011). Y, al igual que el resto de integrantes de dicho consejo, el director de comunicación ha de utilizar indicadores cuantitativos que le permitan evaluar la excelencia y eficacia de las distintas acciones de comunicación institucional que lleva a cabo el hospital (Moreno, Wiesenberg y Verčič, 2016: 13)

El director de comunicación del hospital debe identificar los diez stakeholders principales con los que interactúa el profesional de la salud en las redes sociales. Para cada uno de ellos, tiene que fijar un objetivo de comunicación, así como un valor de marca —tangible o intangible-que el profesional de la salud ha de transmitir cuando interactúa con ellos en las redes sociales. De este modo, el profesional de la salud adapta su comunicación según estos dos indicadores (objetivo de comunicación y valor de marca). A continuación, se analiza el Modelo PMA de Medical Branding para crear valor de marca en las redes sociales a través de la comunicación interpersonal que mantiene el profesional de la salud con los distintos stakeholders. Este modelo propone diez stakeholders, y para cada uno de ellos un objetivo comunicativo y un valor de marca (véase Tabla 1. Modelo PMA de Medical Branding). El modelo presentado en este artículo constituye una continuación del Modelo PMA de reputación online hospitalaria, el cual propone diez fases que ayudan a estas organizaciones a mejorar su imagen en las redes sociales (Medina, 2017a). A continuación, se explican los diez stakeholders, objetivos y valores del modelo PMA de Medical Branding.

1. Empleados del hospital. El recurso a las redes sociales como herramienta de comunicación interna entre los empleados es cada vez más frecuente. Gracias a dichas plataformas, los profesionales de la salud pueden compartir información con el resto de empleados, lo que resulta fundamental para ofrecer al paciente un servicio médico integral (Rodrigues, Azevedo y Calvo, 2016). Por eso, resulta necesario que dichos profesionales fomenten el valor del compromiso organizacional, de tal modo que todos los empleados colaboren en la consecución de los objetivos globales perseguidos por el hospital.

2. Personal técnico externo al hospital. Los hospitales colaboran con diferentes proveedores sanitarios (ambulancias, centros de día, asistentes sociales, etc.), con quienes resulta fundamental establecer una coordinación que permita garantizar la calidad de los servicios médicos ofrecidos al paciente. Los profesionales de la salud que recurren a las redes sociales para comunicarse con el personal técnico externo a la organización deben priorizar el valor del respeto a la cultura corporativa del hospital, para así fomentar la percepción única de la marca hospitalaria por parte del paciente. La promoción de la cultura corporativa a través de las redes sociales influye positivamente en la reputación de la marca hospitalaria (Helfert et al., 2013).

3. Médicos e investigadores externos. Los profesionales de la salud recurren a redes sociales, como Twitter y LinkedIn, para ponerse en contacto con médicos e investigadores que trabajan en otros centros hospitalarios, ya sea a escala nacional o internacional (Antheunis, Tates y Nieboer, 2013). El objetivo de 
dicha comunicación es compartir información científica, consultar dudas, así como promocionar la marca personal. Cuando los profesionales de la salud interactúan con este público a través de las redes sociales, el principal valor de marca que deben transmitir es el sentimiento de pertenencia a la organización hospitalaria.

4. Paciente que acude al centro hospitalario. Gracias a las redes sociales, los profesionales de la salud pueden comunicarse con el paciente antes, durante y después de la consulta y formarles en temas de prevención y educación sanitaria. Esta comunicación constante permite al profesional de la salud convertirse en una fuente de información médica, lo que significa que el principal valor de marca que este debe promocionar en las redes sociales cuando interactúa con los pacientes es la credibilidad científica. A menudo, los pacientes que acuden a las redes sociales para buscar información médica se quejan de la falta de precisión de la misma e incluso de la falta de elementos informativos necesarios para comprender sus problemas de salud (Dijs et al., 2010); de ahí la importancia de que el profesional de la salud desarrolle su credibilidad como educador en temas médicos.

5. Asociaciones de pacientes y de profesionales sanitarios. Los profesionales de la salud pueden utilizar las redes sociales para comunicarse con las asociaciones de pacientes y de profesionales sanitarios para transmitirles información sobre patologías, tratamientos, prevención, investigación, organización sanitaria, acceso a los sistemas de salud, consultoría legal, etc. El principal objetivo de esta comunicación es el acompañamiento constante de los potenciales pacientes y de los profesionales de la salud — que pueden colaborar con el centro hospitalario en un momento dado-; por eso, el principal valor de marca que debe transmitir el profesional de la salud es el altruismo. Para cambiar el comportamiento sanitario de la población, dichos profesionales tienen que conocer bien las necesidades, barreras y oportunidades que tienen los pacientes, y mostrarles siempre un compromiso sincero con ellos (Jones et al., 2015).

6. Centros de formación. Los profesionales de la salud están en contacto con profesores y alumnos de distintos centros de formación, como las universidades y los centros de formación profesional. Cuando dichos profesionales utilizan las redes sociales para comunicarse con este público deben priorizar, como objetivo principal, la transmisión de pasión por las profesiones de salud, y, como valor de marca, la apuesta constante por la innovación como método para satisfacer al paciente. Las universidades tienen que fomentar la formación de los futuros profesionales de la salud en ámbitos como la comunicación, la innovación y el servicio integral al paciente (Gilligan et al., 2016).

7. Medios de comunicación. A menudo, los periodistas contactan a los profesionales de la salud sin pasar previamente por el departamento de comunicación del hospital. Esta situación supone un riesgo comunicativo para la marca hospitalaria. Las relaciones comunicativas que los profesionales de la salud mantienen con los periodistas en las redes sociales deben perseguir un objetivo único: difundir información institucional que satisfaga las necesidades informativas de los periodistas. El principal valor de marca que han de 
potenciar es la experiencia científica. Transmitir información precisa y actualizada influye positivamente en la imagen que los medios de comunicación construyen sobre las organizaciones hospitalarias (Gurau, 2013).

8. Autoridades sanitarias. Los profesionales de la salud interactúan con distintos integrantes de las autoridades sanitarias, ya sean estas regionales, nacionales o internacionales. A menudo, dichas autoridades solicitan la colaboración de los profesionales de la salud para proyectos de investigación, campañas de prevención, evaluación de informes, etc. En las redes sociales, los profesionales de la salud deben transmitir a dichas autoridades informaciones científicas e institucionales que permitan realzar el valor de marca del hospital. Los hospitales tienen que adoptar un enfoque más estratégico a la hora de gestionar su imagen en las redes sociales para así mejorar la reputación de la organización (Park, Rodgers y Stemmle, 2013); por eso, el valor de marca que han de transmitir los profesionales de la salud cuando interactúan con las autoridades sanitarias es la responsabilidad social.

9. Laboratorios farmacéuticos. La reputación de la marca hospitalaria depende de la reputación de los profesionales de la salud que trabajan en la organización (Torres, Vásquez y Barra, 2009), por tanto, resulta importante que cuando dichos profesionales interactúan en las redes sociales con integrantes de la industria farmacéutica sean muy prudentes y orienten dicha comunicación a la consecución de un único objetivo: comprender mejor los tratamientos farmacéuticos que podrían mejorar la calidad de vida del paciente. Los profesionales de la salud deben transmitir como único valor de marca la fidelidad al paciente.

10. Compañías de seguros. Los hospitales tienen que asumir la responsabilidad de situar al paciente en el centro del funcionamiento de la organización sanitaria, lo que implica que han de ayudarle en las gestiones que este realiza con los proveedores externos, como por ejemplo las compañías de seguros (Wynia y Osborn, 2010). Los profesionales de la salud que interactúan en las redes sociales con dichas compañías de seguros deben proteger siempre los intereses del paciente y de sus familiares. El principal valor de marca que tienen que transmitir es el servicio integral ofrecido al paciente.

Tabla 1. Modelo PMA de Medical Branding

\begin{tabular}{l|l|l|l} 
& Stakeholder & Objetivo comunicativo & Valor de marca \\
\hline 1 & Empleados del hospital & Compartir información & Compromiso organizacional \\
\hline 2 & $\begin{array}{l}\text { Personal técnico externo } \\
\text { al hospital }\end{array}$ & Coordinar servicios & $\begin{array}{l}\text { Respeto de la cultura } \\
\text { corporativa del hospital }\end{array}$ \\
\hline 3 & $\begin{array}{l}\text { Médicos e investigadores } \\
\text { externos }\end{array}$ & $\begin{array}{l}\text { Difundir información, } \\
\text { solucionar dudas, personal } \\
\text { branding }\end{array}$ & $\begin{array}{l}\text { Sentimiento de pertenencia al } \\
\text { hospital }\end{array}$ \\
\hline 4 & Paciente & Educar y prevenir & Credibilidad científica \\
\hline 5 & $\begin{array}{l}\text { Asociaciones de pacientes y de } \\
\text { profesionales sanitarios }\end{array}$ & Acompañar & Altruismo \\
\hline
\end{tabular}




\begin{tabular}{l|l|l|l} 
& Stakeholder & Objetivo comunicativo & Valor de marca \\
\hline 6 & Centros de formación & $\begin{array}{l}\text { Transmitir pasión por la } \\
\text { profesión }\end{array}$ & Innovación constante \\
\hline 7 & Medios de comunicación & $\begin{array}{l}\text { Difundir información } \\
\text { institucional }\end{array}$ & Experiencia científica \\
\hline 8 & Autoridades sanitarias & $\begin{array}{l}\text { Difundir información científica } \\
\text { e institucional }\end{array}$ & Responsabilidad social \\
\hline 10 & Compañías de seguros & $\begin{array}{l}\text { Comprender los nuevos } \\
\text { tratamientos farmacéuticos }\end{array}$ & Fidelidad al paciente \\
\hline Proteger los intereses del & Servicio integral al paciente
\end{tabular}

La aplicación del Modelo PMA de Medical Branding aporta varias ventajas a las organizaciones hospitalarias. Por un lado, permite estructurar la comunicación en redes sociales de un modo sencillo y estratégico; y, por otro, convierte al profesional de la salud en un verdadero embajador de la marca hospitalaria, lo que influye positivamente en la credibilidad y reputación de la misma. La aplicación de este modelo implica que el departamento de comunicación del hospital debe evaluar constantemente las conversaciones mantenidas por los profesionales de la salud para analizar así el impacto de cada conversación en la reputación personal del profesional de la salud, así como en la reputación de la marca hospitalaria (McCaughey et al., 2014).

A pesar del interés de este estudio, se pueden señalar varias limitaciones, como por ejemplo la ausencia de cifras cuantitativas sobre el uso de las redes sociales por parte de los hospitales, la dificultad para acceder a las estrategias de comunicación online seguidas por dichas organizaciones y la dificultad para obtener datos sobre las percepciones que tienen los pacientes sobre la comunicación online mantenida con el profesional de la salud. Sería interesante que, en los próximos años, otros investigadores pudieran analizar temas relacionados con la formación del profesional de la salud en habilidades de comunicación online, las estrategias de comunicación interpersonal para divulgar la marca hospitalaria o las estrategias de comunicación para gestionar las percepciones de los pacientes.

\section{CONCLUSIONES}

La implementación de las redes sociales como herramienta de comunicación institucional, las nuevas exigencias de los pacientes, su interés marcado por la educación sanitaria y la prevención, así como la necesidad de los hospitales de crear marcas sólidas y reputadas, han llevado a los profesionales de la salud a asumir nuevas responsabilidades comunicativas. Dichos profesionales están llamados a convertirse en los principales comunicadores del hospital. Así, a las funciones habituales que estos ya asumen (asistencia hospitalaria, investigación y docencia), 
se añade una más: convertirse en embajadores de la marca hospitalaria. Los hospitales tienen que cambiar sus estrategias de comunicación tradicionalmente centradas en herramientas unidireccionales (prensa, revistas corporativas, newsletters, etc.) e instaurar estrategias de comunicación institucional centradas en el protagonismo del profesional de la salud, la apuesta prioritaria por las redes sociales, la difusión de contenido con valor añadido y la promoción de la marca corporativa. Y, para ello, dichas organizaciones necesitan tomar tres medidas. En primer lugar, la dirección del hospital debe realizar los cambios económicos y de management que sean necesarios para que los profesionales de la salud dispongan del tiempo necesario para formarse en comunicación institucional y para interactuar en las redes sociales con los distintos stakeholders. En segundo lugar, el director de comunicación del hospital ha de diseñar un plan de comunicación institucional -interpersonal, interna y externa- basado en protocolos concretos e indicadores de evaluación que le permitan analizar el impacto de la comunicación en la reputación de la marca hospitalaria. Y, en tercer lugar, los profesionales de la salud y de la comunicación tienen que trabajar en estrecha colaboración para elaborar contenidos científicos que aporten valor a la marca hospitalaria y que a su vez puedan ser difundidos en redes sociales a los distintos stakeholders.

Pablo Medina Aguerrebere (pmpelz@ hotmail.com) es doctor en Comunicación Institucional (Universidad de Navarra, 2011). Actualmente trabaja en la Canadian University Dubai, como profesor en la Facultad de
Comunicación, donde realiza labores de investigación sobre el área de comunicación de salud. Sus principales ámbitos de docencia son la comunicación institucional, el management en las agencias de publicidad y el marketing.

\section{Bibliografía}

Abramson, K.; Keefe, B. y Chou, W. (2015). "Communicating About Cancer Through Facebook: A Qualitative Analysis of a Breast Cancer Awareness Page". Journal of Health Communication, 20(2), pp. 237-243.

Al-Abri, R. y Al-Balushi, A. (2014). "Patient Satisfaction Survey As a Tool Towards Quality Improvement". Oman Medical Journal, 29(1), pp. 3-7.

Antheunis, M.; Tates, K. y Nieboer, T. (2013). "Patients' and Health Professionals' Use of Social Media in Healthcare: Motives,
Barriers and Expectations". Patient Education and Counselling, 92, pp. 426-431.

Blomgren, M.; Hedmo, T. y Waks, C. (2016). "Being Special in an Ordinary Way: Swedish Hospitals' Strategic Web Communication". International Journal of Strategic Communication, 10(3), pp. 177-194.

Bourke, R. (2009). An Investigation into the Reputation of a Public Hospital. Waterford, Ireland: Waterford Institute of Technology.

Bubien, Y. (2015). "Hôpital 2.0: du virtuel au réel". European Psychiatry, 30(8), pp. S74. 
Cady, S.; Wheeler, J.; DeWolf, J. y Brodke, M. (2011). "Mission, Vision and Values: What Do They Say?" Organizational Development Journal, 29(1), pp. 63-78.

Chretien, K. y Terry, K. (2013). "Social Media and Clinical Care. Ethical, Professional and Social Implications". Circulation, 127, pp. 1413-1421.

Dijs, J.; Otten, W.; Versluijs, M.; Smeets, H. y Kievit, J. (2010). "Choosing a Hospital for Surgery: The Importance of Information on Quality of Care". Medical Decision Making, 30(5), pp. 544-55.

Esposito, A. (2017). "Hospital Branding in Italy: A Pilot Study Based on the Case Method". Health Marketing Quarterly, 34(1), pp. 35-47.

Farnan, J. M.; Snyder, S. L.; Worster, B. K.; Chaudhry, H. J.; Rhyne, J. A. y Arora, V. M. (2013). “Online Medical Professionalism: Patient and Public Relationships: Policy Statement from the American College of Physicians and the Federation of State Medical Boards". Annals International of Medicine, 158(8), pp. 620-627.

Fernández, L. y Bau, T. (2015). "Health and Social Media: Perfect Storm of Information". Healthcare Informatics Research, 21(2), pp. 67-73.

Gabarron, E.; Fernández, L.; Armayones, M. y Lau, A. (2013). "Identifying Measures Used for Assessing Quality of YouTube Videos with Patient Health Information: A Review of Current Literature". Interactive Journal of Medical Research, 2(1), pp. e6. DOI:10.2196/ ijmr.2465.

Gay, S. y Pho, K. (2013). “Online Reputation Management: The First Steps". The Journal of Medical Practice Management, 29(2), pp. 81-83.

Gilligan, C.; James, E. L.; Snow, P.; Outram, S.; Ward, B. M.; Powell, M.; Lonsdale, C.; Cushing, A. M.; Silverman, J.; Regan, T.; Harvey, P. y Lynagh, M. C. (2016). "Interventions for Improving Medical Students' Interpersonal
Communication in Medical Consultations". Cochrane Database of Systematic Reviews, 11.

Grajales, F.; Sheps, S.; Kendall, H.; Novak, H. y Eysenbach, G. (2014). "Social Media: A Review and Tutorial of Applications in Medicine and Health Care". Journal of Medical Internet Research, 16(2), pp. e13.

Griffis, H.; Kilaru, A.; Werner, R.; Asch, D.; Hershey, J.; Hill, S.; Ha, Y.; Sellers, A.; Mahoney, K. y Merchant, R. (2014). "Use of Social Media Across US Hospitals: Descriptive Analysis of Adoption and Utilization". Journal of Medical Internet Research, 16(11), pp. e264.

Gurau, C. (2013). "Developing an Environmental Corporate Reputation on the Internet". Marketing Intelligence \& Planning, 31(5), pp. 522-537.

Hannawa, A.; García, L.; Candrian, C.; Rossmann, C. y Schulz, P. (2015). "Identifying the Field of Health Communication". Journal of Health Communication, 20(5), pp. 521-530.

Helfert, M.; Lugmayr, A.; Heimgärtner, R. y Holzinger, A. (2013). "Influence of Organizational Culture and Communication on the Successful Implementation of Information Technology in Hospitals". Human Computer Interaction, vol. 21, pp. 165-174.

Jones, C.; Jensen, J.; Scherr, C.; Brown, N.; Christy, K. y Weaver, J. (2015). “The Health Belief Model As an Explanatory Framework in Communication Research Exploring Parallel, Serial, and Moderated Mediation". Health Communication, 30(6), pp. 566-576.

Kemp, E.; Jillapalli, R. y Becerra, E. (2014). "Healthcare Branding: Developing Emotionally Based Consumer Brand Relationships". Journal of Services Marketing, 28(2), pp. 126-137.

Lim, W. (2016). "Social Media in Medical and Health Care: Opportunities and Challenges". Marketing Intelligence \& Planning, 34(7), pp. 964-976.

Maier, C. (2016). "Beyond Branding: Van Riel and Fombrun's Corporate Communication Theory in the Human Services Sector". 
22 Qualitative Research Reports in Communication, 17(1), pp. 27-35.

McCarroll, M.; Armbruster, S.; Chung, J.; Kim, J.; McKenzie, A. y Gruenigen, V. von (2014). "Health Care and Social Media Platforms in Hospitals". Health Communication, 29(9), pp. 947-952.

McCaughey, D.; Baumgardner, C.; Gaudes, A.; LaRochelle, D.; Wu, K. y Raichura, T. (2014). "Best Practices in Social Media: Utilizing a Value Matrix to Assess Social Media's Impact on Health Care". Social Science Computer Review, 32(5), pp. 575-589.

Medina, P. (2011). Organización de la comunicación interna. Madrid: Fragua. ISBN: 8470744453.

—. (2017a). "La gestión de la reputación 'online' de las marcas hospitalarias: una propuesta de modelo". Zer, 22, pp. 53-68.

—. (2017b). "L'impact des réseaux sociaux sur la communication hospitalière aux Etats-Unis". Revue Française des Sciences de l'Information et de la Communication, 11.

Mira, J.; Lorenzo, S. y Navarro, I. (2014). "Hospital Reputation and Perceptions of Patient Safety". Medical Principles and Practice, 23, pp. 92-94.

Moreno, A.; Wiesenberg, M. y Verčič, D. (2016). Excelencia en la Gestión de Comunicación. Análisis de los Departamentos de comunicación en España mediante el Comparative Excellence Framework. Comhumanitas: Revista Científica de Comunicación, 7(2), pp. 1-15.

Moser, R. y Greeman, G. (2014). “An Empirical Analysis of the Public's Attitudes Toward Advertising Hospital Services: A Comparative Cross-Sectional Study". Health Marketing Quarterly, 31, pp. 13-30.

Nelson, W.; Taylor, E. y Walsh, T. (2014). "Building an Ethical Organizational Culture". The Health Care Manager, 33(2), pp. 158-164.

Park, H.; Rodgers, S. y Stemmle, J. (2013). “Analyzing Health Organizations' Use of Twitter for Promoting Health Literacy". Journal of Health Communication, 18(4), pp. 410-425.
Pelitti, P. (2016). "Estrategias de comunicación interna y externa de los hospitales públicos bonaerenses de la Región Sanitaria X". Question. Revista especializada en comunicación $y$ periodismo, 1(49), pp. 368-379.

Rando, D. y Heras, C. de las (2016). "Análisis de la comunicación corporativa de los hospitales andaluces vía Twitter". Opción, 32(8), pp. 557-576.

Rodrigues, A.; Azevedo, C. y Calvo, V. (2016). "Internal Communication in Organizations: Practical Instruments to Help the Shift Change". Millenium, 2(1), pp. 105-114.

Ruiz, M. (2015). "Análisis comunicacional de páginas web hospitalarias. El caso de los hospitales sevillanos". Revista Española de Comunicación y Salud, 6(2), pp. 138-56.

Sheehan, N. y Isaac, G. (2014). "Principles Operationalize Corporate Values so They Matter". Strategy \& Leadership, 42(3), pp. 23-30.

Singal, A. y Jain, A. (2013). "An Empirical Examination of the Influence of Corporate Vision on Internationalization". Strategic Change, 22(5-6), pp. 243-257.

Torres, E.; Vásquez, A. y Barra, C. (2009). "The Path of Patient Loyalty and the Role of Doctor Reputation". Health Marketing Quarterly, 26(3), pp. 183-197.

Trepanier, S. y Gooch, P. (2014). "Personal Branding and Nurse Leader Professional Image". Nurse Leader, 12(3), pp. 51-57.

Veltri, S. y Nardo, M. (2013). "The Intangible Global Report: An Integrated Corporate Communication Framework". Corporate Communications: An International Journal, 18(1), pp. 26-51.

Wang, Y.; Hsu, K.; Hsu, S. y Hsieh, P. (2011). "Constructing an Index for Brand Equity: A Hospital Example". The Service Industries Journal, 31(2), pp. 311-322.

Weech, R.; Elliott, M.; Pradhan, R.; Schiller, C.; Hall, A. y Hays, R. (2012). "Can Hospital Cultural Competency Reduce Disparities in Patient Experiences with Care". Medical Care, 50, pp. S48-S55. 
Würz, A.; Nurm, Ü. y Ekdahl, K. (2013). "Enhancing the Role of Health Communication in the Prevention of Infectious Diseases". Journal of Health Communication, 18(12), pp. 1566-1571.

Wynia, M. y Osborn, C. (2010). "Health Literacy and Communication Quality in
Health Care Organizations". Journal of Health Communication, 15(S2), pp. 102-115.

Zerfass, A. y Viertmann, C. (2017). "Creating Business Value Through Corporate Communication: A Theory-Based Framework and Its Practical Application". Journal of Communication Management, 21(1), pp. 68-81. 
